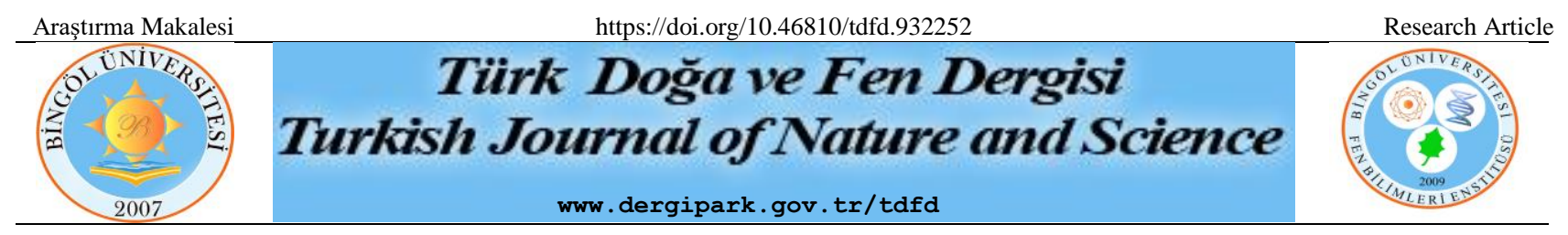

Investigation of Nonlinear Wave Solutions in Fluid Mechanics

\author{
Tolga AKTÜRK ${ }^{*}$, Yusuf GUREFE ${ }^{2}$ \\ ${ }^{1}$ Ordu University, Faculty of Education, Department of Mathematics and Science Education, Ordu, Turkey \\ ${ }^{2}$ Mersin University, Faculty of Sciences, Department of Mathematics, Mersin, Turkey \\ Tolga AKTÜRK ORCID No: 0000-0002-8873-0424 \\ Yusuf GUREFE ORCID No: 0000-0002-7210-5683
}

*Corresponding author: tolgaakturk@odu.edu.tr

(Alınış: 03.05.2021, Kabul: 18.09.2021, Online Yayınlanma: 31.12.2021)

\author{
Keywords \\ Modified \\ exponential \\ function method, \\ The $(3+1)$ - \\ dimensional $\mathrm{Yu}-$ \\ Toda-Sasa- \\ Fukuyama \\ equation, \\ Traveling wave \\ solutions
}

\begin{abstract}
In this study, the traveling wave solutions of the $(3+1)$-dimensional potential Yu-Toda-Sasa-Fukuyama (YTSF) equation are get using the modified exponential function method (MEFM). It has been observed that the obtained solution functions are in the form of trigonometric, hyperbolic and rational functions. The solution functions that arose from the process of the method are checked by Wolfram Mathematica software and it has seen that they satisfy the $(3+1)$ - dimensional potential Yu-Toda-Sasa-Fukuyama (YTSF) equation. Two and three dimensional, contour and density graphs of the solution function are found by determining the appropriate parameters.
\end{abstract}

\title{
Akışkanlar Mekaniğinde Doğrusal Olmayan Dalga Çözümlerinin İncelenmesi
}

\author{
Anahtar Kelimeler \\ Geliştirilmiş üstel \\ fonksiyon metodu, \\ (3+1)-Boyutlu Yu- \\ Toda-Sasa- \\ Fukuyama \\ denklemi, \\ Hareketli dalga \\ çözümleri
}

Öz: $\mathrm{Bu}$ çalışmada, $(3+1)$ boyutlu potansiyel Yu-Toda-Sasa-Fukuyama (YTSF) denkleminin yürüyen dalga çözümleri değiştirilmiş üstel fonksiyon yöntemi (MEFM) kullanılarak elde edilmiştir. Elde edilen çözüm fonksiyonlarının trigonometrik, hiperbolik ve rasyonel fonksiyonlar şeklinde olduğu görülmüştür. Yöntemin işleminde ortaya çıkan çözüm fonksiyonları Wolfram Mathematica yazılımı ile kontrol edilmiş ve $(3+1)$ boyutlu potansiyel Yu-Toda-Sasa-Fukuyama (YTSF) denklemini sağladıkları görülmüştür. Çözüm fonksiyonunun iki ve üç boyutlu, kontur ve yoğunluk grafikleri uygun parametreler belirlenerek bulunur.

\section{INTRODUCTION}

Nonlinear partial differential equations and the solutions of these types of equations are used in physics, engineering, health, and social sciences, etc. They have an essential place in the branches of science because such equations represent the mathematical model of a given event. In the literature, there are various methods for obtaning the numerical or analytical solutions of such equations. Some of these methods in the literatüre are, the generalized Bernoulli sub-equation function method [1-2], the trial equation method [3-7], the modified, extended tanh-function method [8-9], the first integral method [10], generalized tanh function method [11], the modified exponential function method [12-16] and many more methods.
In this study, we consider the $(3+1)$ dimensional potential YTSF equation given in the following [17-19],

$$
-4 u_{x t}+u_{x x x z}+4 u_{x} u_{x z}+2 u_{x x} u_{z}+3 u_{y y}=0 .
$$

The mathematical model in equation (1) has an important place in plasma physics and fluid dynamics. There are various methods in the literature [20-25] for the solutions of equation (1). In the second part of this study, the modified exponential function method is introduced. In the third part, the solutions for the $(3+1)$ dimensional YTSF equation are obtained by applying MEFM. Besides that two and three dimensional and contour graphs are presented. The conclusion part is given in the end. 


\section{MATERIALS AND METHODS}

Let us consider the general form of the nonlinear partial differential equation for the modified exponential function method as follows;

$$
P\left(U, U_{x}, U_{y}, U_{z}, U_{t}, U_{x x}, U_{x t}, U_{y y}, U_{x x x z}, \cdots\right)=0,
$$

where $U=U(x, y, z, t)$ is the unknown function.

Step 1. The wave transformation given below is considered for the independent variables of equation (1),

$$
U(x, y, z, t)=U(\xi), \xi=k(x+y+z-c t),
$$

The terms $k$ and $c$ in the wave transformation are constants. If the solution function $U(\xi)$ in (3) and the related derivatives are substituted into (2), a nonlinear ordinary differential equation is obtained as in the following form,

$$
N\left(U, U^{\prime},\left(U^{\prime}\right)^{2}, U^{\prime \prime}, U^{\prime \prime \prime}, \cdots\right)=0,
$$

the general form of the nonlinear ordinary differential equation is get.

Step 2: According to this method, the solution function of equation (1) is as follows;

$$
\begin{aligned}
& U(\xi)=\frac{\sum_{i=0}^{n} A_{i}[\exp (-\Omega(\xi))]^{i}}{\sum_{j=0}^{m} B_{j}[\exp (-\Omega(\xi))]^{j}}= \\
& =\frac{A_{0}+A_{1} \exp (-\Omega)+\cdots+A_{n} \exp (n(-\Omega))}{B_{0}+B_{1} \exp (-\Omega)+\cdots+B_{m} \exp (m(-\Omega))},
\end{aligned}
$$

where $A_{i}, B_{j},(0 \leq i \leq n, 0 \leq j \leq m)$ are constants. The balancing procedure is applied to the nonlinear ordinary differential equation (4) obtained by using the wave transformation. In other words, by balancing the term having the highest order derivative and the nonlinear term in equation (4), the relation between $m$ and $n$ is obtained. By determining the parameters that satisfy the this balancing relation, the upper limits of the summation symbols in equation (5) are revealed.

The $\Omega(\xi)$ function that situated in (5) satisfies the following ODE [27].

$$
\Omega^{\prime}(\xi)=\exp (-\Omega(\xi))+\mu \exp (\Omega(\xi))+\lambda .
$$

Family 1: When $\mu \neq 0, \lambda^{2}-4 \mu>0$,

$$
\Omega(\xi)=\ln \left(\begin{array}{l}
\frac{-\sqrt{\lambda^{2}-4 \mu}}{2 \mu} \tanh \left(\frac{\sqrt{\lambda^{2}-4 \mu}}{2}(\xi+E)\right)- \\
-\frac{\lambda}{2 \mu}
\end{array}\right) .
$$

Family 2: When $\mu \neq 0, \lambda^{2}-4 \mu<0$,

$$
\Omega(\xi)=\ln \left(\begin{array}{l}
\frac{\sqrt{-\lambda^{2}+4 \mu}}{2 \mu} \tan \left(\frac{\sqrt{-\lambda^{2}+4 \mu}}{2}(\xi+E)\right) \\
-\frac{\lambda}{2 \mu}
\end{array}\right) .
$$

Family 3: When $\mu=0, \lambda \neq 0$ and $\lambda^{2}-4 \mu>0$,

$$
\Omega(\xi)=-\ln \left(\frac{\lambda}{\exp (\lambda(\xi+E))-1}\right)
$$

Family 4: When $\mu \neq 0, \lambda \neq 0$ and $\lambda^{2}-4 \mu=0$,

$$
\Omega(\xi)=\ln \left(-\frac{2 \lambda(\xi+E)+4}{\lambda^{2}(\xi+E)}\right) .
$$

Family 5: When $\mu=0, \lambda=0$ and $\lambda^{2}-4 \mu=0$,

$$
\Omega(\xi)=\ln (\xi+E)
$$

Step 3: The substitution of (5) into NLODE (4), considering (6), produces an algebraic equation system consisting of coefficients $A_{0}, A_{1}, \ldots, A_{n}, B_{0}, B_{1}, \ldots, B_{m}$. When this system of equations is solved with the help of the Mathematica program, the traveling wave solutions that satisfy equation (1) are obtained.

\section{APPLICATIONS}

By using the traveling wave transformation (3), equation (1) return to the following nonlinear ordinary differential equation,

$$
(4 c+3) U^{\prime}+k^{2} U^{\prime \prime \prime}+3 k\left(U^{\prime}\right)^{2}=0 .
$$

If $U^{\prime}=V$ transform is applied in order to make integral operations with ease in equation (12),

$$
(4 c+3) V+k^{2} V^{\prime \prime}+3 k V^{2}=0 .
$$


In equation (13), if the equalization term is applied between $V^{\prime \prime}$ and $V^{2}$ according to the definition given above,

$$
M+2=N .
$$

If $M=1$ is chosen so as to provide the equality in equation (14), $N=3$ is obtained. In this case, the necessary derivative terms in equation (5) and the nonlinear ordinary differential equation are obtained as follows:

$$
\begin{aligned}
& V(\xi)=\frac{\psi}{\varphi}=\frac{A_{0}+A_{1} e^{-\Omega(\xi)}+A_{2} e^{-2 \Omega(\xi)}+A_{3} e^{-3 \Omega(\xi)}}{B_{0}+B_{1} e^{-\Omega(\xi)}}, \\
& V^{\prime}(\xi)=\frac{\psi^{\prime} \varphi-\psi \varphi^{\prime}}{\varphi^{2}}, \\
& V^{\prime \prime}(\xi)=\frac{\psi^{\prime \prime} \varphi^{3}-\varphi^{2} \psi^{\prime} \varphi^{\prime}-\left(\psi \varphi^{\prime \prime}+\psi^{\prime} \varphi^{\prime}\right) \varphi^{2}+2\left(\psi^{\prime}\right)^{2} \psi \varphi}{\varphi^{4}} .
\end{aligned}
$$

\section{CASE 1:}

$$
\begin{gathered}
A_{0}=\frac{\lambda^{2} A_{3} B_{0}}{4 B_{1}}+\frac{(3+4 c) B_{0} B_{1}}{A_{3}}, \\
A_{1}=\frac{1}{4} \lambda A_{3}\left(\lambda+\frac{4 B_{0}}{B_{1}}\right)+\frac{(3+4 c) B_{1}^{2}}{A_{3}}, \\
A_{2}=A_{3}\left(\lambda+\frac{4 B_{0}}{B_{1}}\right), k=-\frac{A_{3}}{2 B_{1}}, \\
\mu=\frac{\lambda^{2}}{4}+\frac{(3+4 c) B_{1}^{2}}{A_{3}^{2}} .
\end{gathered}
$$

Using the obtained coefficients, let's investigate the traveling wave solutions of equation (1), considering the following family cases.

\section{Family-1:}

$$
V_{1,1}(\xi)=\frac{\left(\operatorname{Sech}\left[\frac{1}{2} \phi\right]^{2}\left(\begin{array}{l}
\left.\left(\sqrt{\lambda^{2}-4 \mu}\right)(-2 \mu+\alpha+\lambda \beta) A_{3}^{2}-\right) \\
-4(3+4 c)(-2 \mu-\alpha-\lambda \beta) B_{1}^{2}
\end{array}\right)\right)}{\left(4 A _ { 3 } B _ { 1 } \left(\lambda+\sqrt{\left.\left.\lambda^{2}-4 \mu \operatorname{Tanh}\left[\frac{1}{2} \phi\right]\right)^{2}\right)}\right.\right.} .
$$

Where $\alpha=\left(\lambda^{2}-2 \mu\right) \operatorname{Cosh}[\phi], \beta=\sqrt{\lambda^{2}-4 \mu} \operatorname{Sinh}[\phi]$, $\phi=\sqrt{\lambda^{2}-4 \mu}(E E+\xi)$.

Integrating both sides of the equation $U^{\prime}=V$ with respect to $\xi$ gives,

$$
\left.U_{1,1}(\xi)=\frac{\left(\begin{array}{l}
\left(\lambda^{2}-4 \mu\right)\left(\begin{array}{l}
-2 \lambda+\lambda^{2}(E E+\xi)- \\
2 \mu(E E+\xi)
\end{array}\right)+ \\
+2\left(\lambda^{2}-4 \mu\right) \mu(E E+\xi) \operatorname{Cosh}[\phi] \\
-4 \sqrt{\lambda^{2}-4 \mu \mu \operatorname{Sinh}[\phi]}
\end{array}\right) A_{3}}{\left(4\left(\lambda^{2}-2 \mu+2 \mu \operatorname{Cosh}[\phi]\right) B_{1}\right)}\right) .
$$

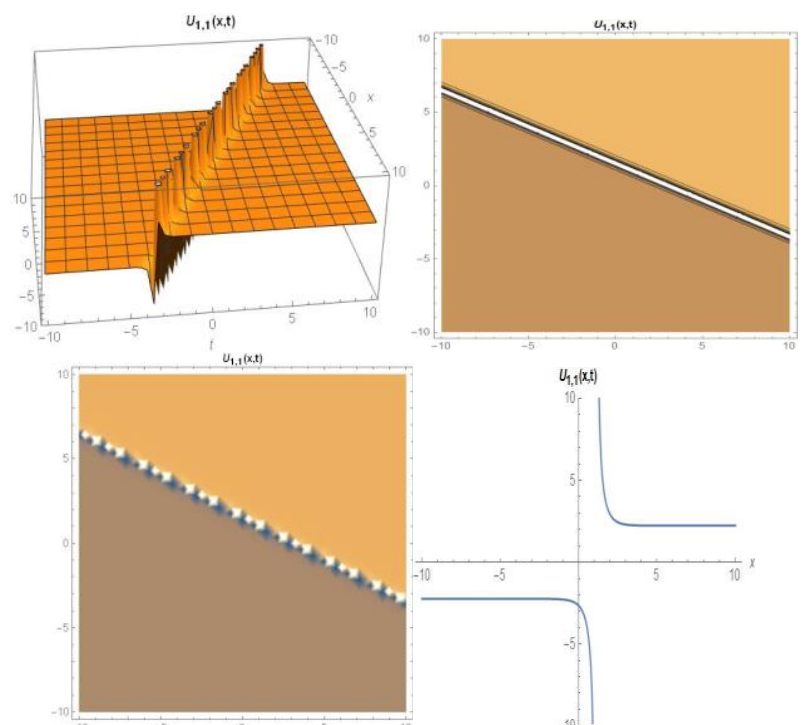

Figure 1. Three-dimensional, contour and density plots of solution (17) for the values $A_{3}=1, c=-2, B_{1}=2, \lambda=1, k=-\frac{1}{4}, \mu=-\frac{79}{4}$, $y=-1, z=1, E E=0.75$ and $t=1$ for the two-dimensional graph.

Family 2:

$$
V_{1,2}(\xi)=-\frac{\left(\left(\operatorname{Sec}\left[\begin{array}{c}
1 \\
-\tau
\end{array}\right]^{2}\left(\begin{array}{l}
\left(\lambda^{2}-4 \mu\right)(2 \mu-\omega+\varsigma) A_{3}^{2}+ \\
4(3+4 c)(-2 \mu-\omega+\varsigma) B_{1}^{2}
\end{array}\right)\right)\right.}{\left.\left(4 A_{3} B_{1}\left(\lambda-\sqrt{-\lambda^{2}+4 \mu \operatorname{Tan}\left[\frac{1}{2} \tau\right.}\right]\right)^{2}\right)}
$$

Where $\varsigma=\lambda \sqrt{-\lambda^{2}+4 \mu} \operatorname{Sin}[\tau], \omega=\left(\lambda^{2}-2 \mu\right) \operatorname{Cos}[\tau]$, $\tau=\sqrt{-\lambda^{2}+4 \mu}(E E+\xi)$.

The solution $U_{1,2}(\xi)$ is obtained by integrating the function $V_{1,2}(\xi)$ with respect to $\xi$. 


$$
U_{1,2}(\xi)=\frac{\left(\begin{array}{l}
\left(\lambda^{2}-4 \mu\right)\left(-2 \lambda+\lambda^{2}(E E+\xi)\right)+ \\
2\left(\left(\lambda^{2}-4 \mu\right) \mu(E E+\xi) \operatorname{Cos}[\tau]+\frac{4 \mu \zeta}{\lambda}\right) A_{3}
\end{array}\right)}{\left(4\left(\lambda^{2}-2 \mu+2 \mu \operatorname{Cos}[\tau]\right) B_{1}\right)+} .
$$
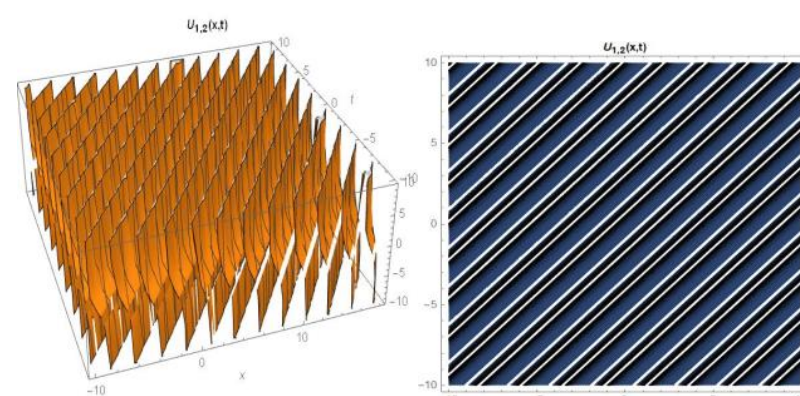

$u_{1,2}(x, t)$

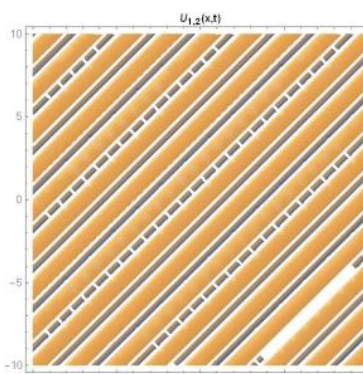

Figure 2. Three-dimensional, contour and density plots of solution (19) for the values $A_{3}=20, c=1, B_{1}=2, \lambda=1, k=-5, \mu=\frac{8}{25}$, $y=-1, z=1, E E=0.75$ and $t=1$ for the two-dimensional graph.

\section{Family 3:}

$V_{1,3}(\xi)=\frac{\lambda^{2} \operatorname{Coth}\left[\frac{1}{2} \lambda(E E+\xi)\right]^{2} A_{3}}{4 B_{1}}+\frac{(3+4 c) B_{1}}{A_{3}}$.

Integrating equation (18) with respect to $\xi$, solution $U_{1,3}(\xi)$ is derived as in the following,

$$
\begin{aligned}
U_{1,3}(\xi) & =\frac{\left(\operatorname{ArcTanh}\left[\operatorname{Tanh}\left[\frac{1}{2} \lambda(E E+\xi)\right]\right]-\right.}{\operatorname{Coth}\left[\frac{1}{2} \lambda(E E+\xi)\right]} A_{3} \\
& +\frac{(3+4 c) \xi B_{1}}{A_{3}}
\end{aligned}
$$
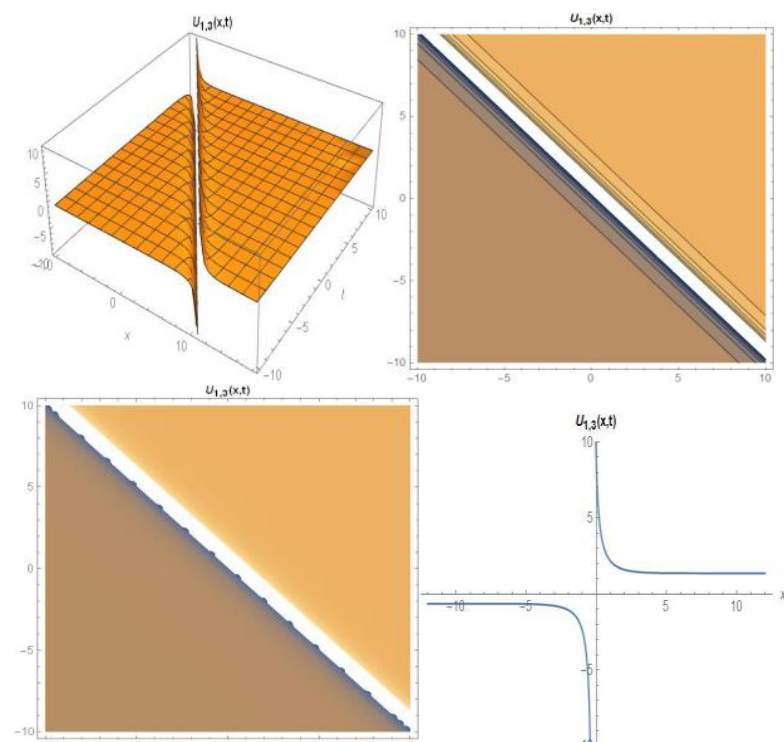

Figure 3. Three-dimensional, contour and density plots of solution (21) for the values $A_{3}=2, c=-1, B_{1}=1, \lambda=1, k=-1, \mu=0$, $y=-1, z=1, E E=0.75$ and $t=1$ for the two-dimensional graph.

\section{Family 4:}

$V_{1,4}(\xi)=\frac{\lambda^{2} A_{3}}{(2+\lambda(E E+\xi))^{2} B_{1}}+\frac{(3+4 c) B_{1}}{A_{3}}$.

Integrating equation (22) with respect to $\xi$, solution $U_{1,4}(\xi)$ is derived as in the following,

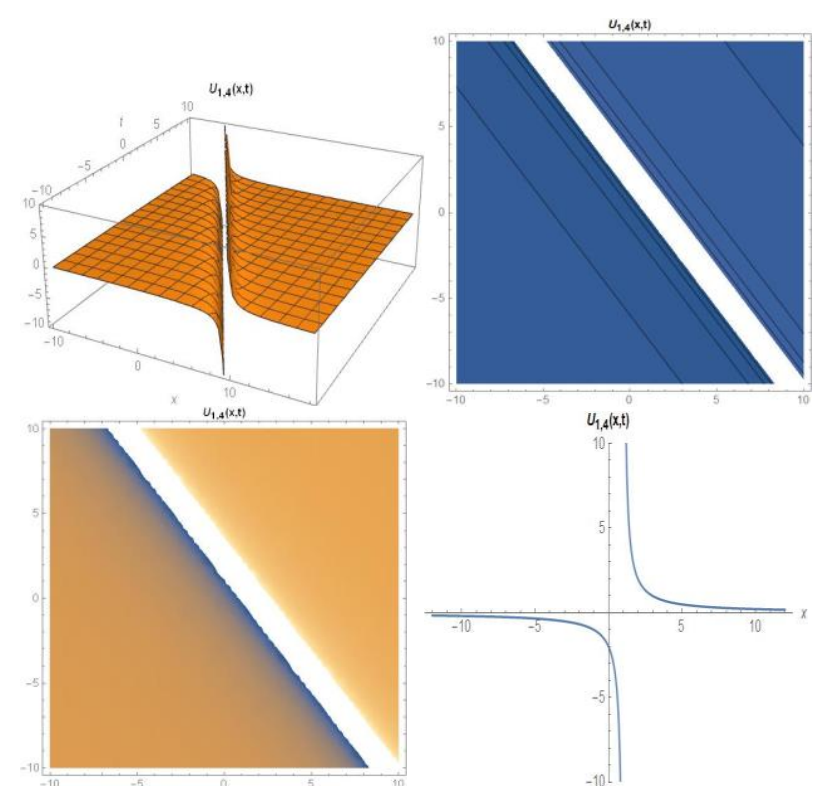

Figure 4. Three-dimensional, contour and density plots of solution (23) for the values $A_{3}=2, c=-\frac{3}{4}, B_{1}=1, \lambda=2, k=-1, \mu=1$, 
$y=-1, z=1, E E=0.75$ and $t=1$ for the two-dimensional graph.

\section{Family 5:}

$V_{1,5}(\xi)=\frac{A_{3}}{(E E+\xi)^{2} B_{1}}+\frac{(3+4 c) B_{1}}{A_{3}}$.

Integrating equation (24) with respect to $\xi$, solution $U_{1,5}(\xi)$ is derived as in the following,

$$
U_{1,5}(\xi)=-\frac{A_{3}}{(E E+\xi) B_{1}}+\frac{(3+4 c) \xi B_{1}}{A_{3}} .
$$

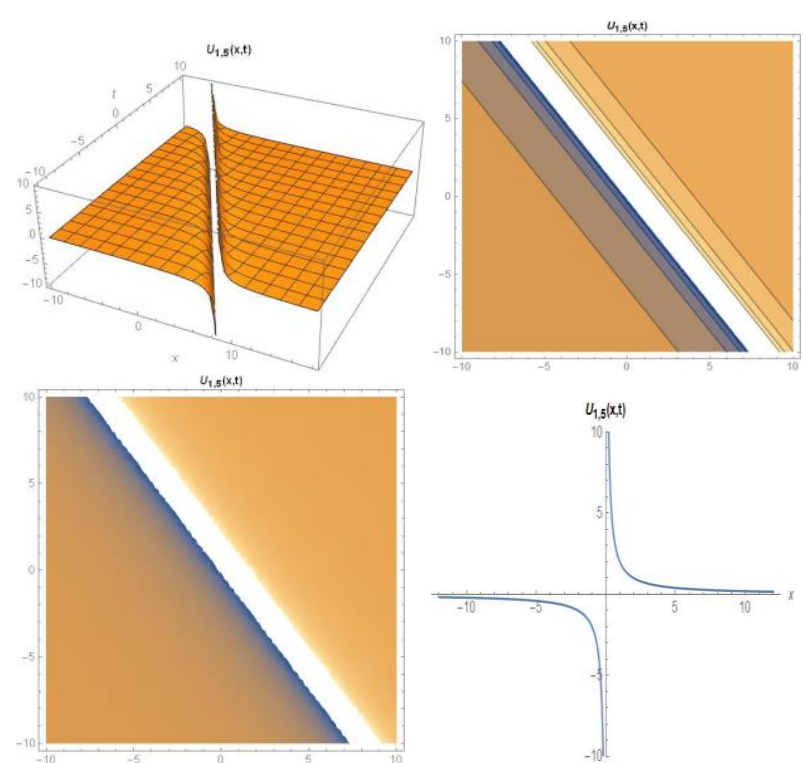

Figure 5. Three-dimensional, contour and density plots of solution (25) for the values $A_{3}=2, c=-\frac{3}{4}, B_{1}=1, \lambda=0, k=-1, \mu=0$ $, y=-1, z=1, E E=0.75$ and $t=1$ for the two-dimensional graph.

\section{CONCLUSION}

In this study, we applied MEFM to the $(3+1)$ dimensional YTSF equation given as a nonlinear mathematical model. In this research, it was determined that all analytical solutions obtained in this study satisfy equation (1). When analytical solution functions are investigated, it is stated that hyperbolic and trigonometric functions have periodic function features and rational functions. All calculations and graphics were obtained using by Mathematica software program. The models of two and three-dimensional graphs remind their physical meaning of traveling wave solutions. If we analyze more situations and take different coefficient values, we can obtain more traveling wave solutions. This MEFM is a reliable technique. The results can help us to learn about the diffusion processes of the nonlinear waves in fluid mechanics.

\section{REFERENCES}

[1] Yang XF, Deng ZC, Wei YA. Riccati-Bernoulli subODE method for nonlinear partial differential equations and its application. Advances in Difference equations. 2015; (1): 1-17.

[2] Baskonus HM, Bulut H. Exponential prototype structures for (2+1)-dimensional Boiti-LeonPempinelli systems in mathematical physics. Waves in Random and Complex Media. 2016; 26(2):189196.

[3] Liu CS. Trial equation method and its applications to nonlinear evolution equations. Acta Physica Sinica. 2005; 54(6): 2505-2509.

[4] Liu CS. Trial equation method to nonlinear evolution equations with rank inhomogeneous: mathematical discussions and its applications. CoTPh. 2006; 45(2): 219-223.

[5] Abdelrahman MA. A note on Riccati-Bernoulli SubODE method combined with complex transform method applied to fractional differential equations. Nonlinear Engineering. 2018; 7(4): 279285.

[6] Bulut H, Baskonus HM and Pandir Y. The modified trial equation method for fractional wave equation and time fractional generalized Burgers equation. In Abstract and Applied Analysis Hindawi. 2013; Vol. 2013.

[7] Gurefe Y, Misirli E, Sonmezoglu A and Ekici M. Extended trial equation method to generalized nonlinear partial differential equations. Applied Mathematics and Computation.2013; 219(10): 5253-5260.

[8] Elwakil SA, El-Labany SK, Zahran MA and Sabry R. Modified extended tanh-function method for solving nonlinear partial differential equations. Physics Letters A. 2002; 299(2-3): 179188.

[9] Fan E and Hon YC. Applications of extended tanh method to 'special'types of nonlinear equations. Applied Mathematics and Computation. 2003; 141(2-3): 351-358.

[10] Hosseini K and Gholamin P. Feng's first integral method for analytic treatment of two higher dimensional nonlinear partial differential equations. Differential Equations and Dynamical Systems. 2015; 23(3): 317-325.

[11]Zheng X, Chen Y and Zhang H. Generalized extended tanh-function method and its application to (1+1)-dimensional dispersive long wave equation. Physics Letters A, 2003; 311(2-3): 145157.

[12] Misirli E and Gurefe Y. The Exp-function method to solve the generalized Burgers-Fisher equation. Nonlinear Science Letter A. 2010; 1: 323328.

[13] Misirli E and Gurefe Y. Exact solutions of the Drinfel'd-Sokolov-Wilson equation using the expfunction method. Applied Mathematics and Computation. 2010; 216(9): 2623-2627.

[14]Baskonus HM and Bulut H. Regarding on the prototype solutions for the nonlinear fractional-order biological population model. In AIP Conference 
Proceedings AIP Publishing LLC. 2016; 1738: 1.

[15] Gurefe Y and Misirli E. Exp-function method for solving nonlinear evolution equations with higher order nonlinearity. Computers \& Mathematics with Applications. 2011; 61(8): 2025-2030.

[16] Özpinar F, Baskonus, HM and Bulut H. On the complex and hyperbolic structures for the $(2+1)$ dimensional boussinesq water equation. Entropy. 2015; 17(12): 8267-8277.

[17] Yan ZY. New families of nontravelling wave solutions to a new $(3+1)$-dimensional potentialYTSF equation. Physics Letters A. 2003; 318: 7883.

[18] Ma WX, Huang T and Zhang Y. A multiple expfunction method for nonlinear differential equations and its application. Physica Scripta. 2010; 82(6): 065003.

[19]Zhang S and Zhang HQ. A transformed rational function method for (3+1)-dimensional potential Yu-Toda-Sasa-Fukuyama equation. Pramana. 2011; 76(4): 561-571.

[20] Roshid HO. Lump solutions to a $(3+1)$-dimensional potential-Yu-Toda-Sasa-Fukuyama (YTSF) like equation. International Journal of Applied and Computational Mathematics. 2017; 3: 1455-1461.

[21]Tan, W. and Dai, Z. Dynamics of kinky wave for (3+1)-dimensional potential Yu-Toda-SasaFukuyama equation. Nonlinear Dynamics. 2016; 85(2): 817-823.

[22]Zayed EME and Ibrahim SH, 2013. The two variable $\left(G^{\prime} / G, 1 / G\right)$-expansion method for finding exact traveling wave solutions of the $(3+1)$ dimensional nonlinear potential Yu-Toda-SasaFukuyama equation. In 2013 International Conference on Advanced Computer Science and Electronics Information (ICACSEI 2013). Atlantis Press.

[23]Zeng XP, Dai ZD, Li DL. New periodic soliton solutions for $(3+1)$-dimensional potential-YTSF equation. Chaos, Solitons Fractals. 2009; 42: 657661.

[24]Zhao $\mathrm{Z}$ and He L. Multiple lump solutions of the (3+1)-dimensional potential Yu-Toda-SasaFukuyama equation. Applied Mathematics Letters. 2019; 95: 114-121.

[25]Dong MJ, Tian, SF, Wang XB and Zhang TT. Lump-type solutions and interaction solutions in the (3+1)-dimensional potential Yu-Toda-SasaFukuyama equation. Analysis and Mathematical Physics. 2019; 9(3): 1511-1523.

[26]Zheng X, Chen Y, Zhang H. Generalized extended tanh-function method and its application to $(1+1)$ dimensional dispersive long wave equation. Physics Letters A. 2003; 311(2-3):145-157.

[27] He $\mathrm{JH}$ and $\mathrm{Wu} \mathrm{XH}$. Exp-function method for nonlinear wave equations. Chaos, Solitons \& Fractals. 2006; 30(3): 700-708. 